\title{
The Method Balance Consistency Availability on Systems Virtual Machine
}

\author{
Saikat Gochhiat, Shariq Aziz Butt, Nguyen ha Huy Cuong.
}

\begin{abstract}
In this time, a few associations are putting away their information on distributed storage so as to meet the prerequisites of effective activity like soundness, versatility, and accessibility of administrations. Information replication benefits in distributed storage frameworks are there to improve execution. In this unique situation, the prerequisites for guaranteeing information consistency have turned out to be progressively significant. In this paper, we have proposed a virtual server solution for refreshing replication, effectively settling assets, distinguishing and avoiding gridlocks at the server sides. In this original study, we have demonstrated that the proposed arrangement yields the outcomes for the construction which guarantees information consistency in regard to expenses and idleness. we utilize the Open Stack apparatus, which joins a proposed calculation on Adjusting Consistency Accessibility in Physical Machine for keeping up information consistency in Distributed storage Frameworks.
\end{abstract}

Keywords: Virtualization, distributed computing, Cloud Storage, Consistency in database systems, Cloud technology.

\section{INTRODUCTION}

$\mathrm{Di}$ stributed storage Frameworks are broadly utilized due to their profoundly proficient reaction to dispersed frameworks. The expanding size of the information is the primary worry of our general public. In this original copy, we have centered to keep up the capacity document proficiently. To limit the time scattered by the client, cloud enables simple organization of administrations to create applications as required by clients based on discrete physical server centres. Data replication is an effective service (response time data, high availability of data, system performance) of the system storage applications in Cloud environments. The reason is that users can go directly to the copy in data centers (Data Center - DC) nearest copy center at the other (an example is shown below) However, data centers have problems such as load balancing, fault tolerance, redundancy, or lack of system resources (memory, processing). Moreover, there are many replicas $(\mathrm{R} 1, \mathrm{R} 2$. . . ) at the physical data centers Figure 1.describes many replicas in cloud storage systems.will make more difficult to ensure data consistency. Thusly, lately, arrangements utilizing virtual server are keen on research, application. That based on the physical server stage can defeat these issues hence improving the effectiveness of the plan to

Revised Manuscript Received on October 31, 2019.

* Correspondence Author

Saikat Gochhait*,Symbiosis Institute of Digital and Telecom Management, constituent of Symbiosis International (Deemed University), Pune, India. Email: saikat.gochhait@sidtm.edu.in

Shariq Aziz Butt, The University of Lahore, Pakistan. Email: shariq2315@gmail.com

Nguyen ha Huy Cuong, The University of Danang, College of Information Technology, Da Nang, Viet Nam. Email: nguyenhahuycuong@gmail.com guarantee information consistency. Every answer for these issues by virtual servers can be considered as a way to deal with improve a proficiency consistency support conspire for distributed storage frameworks. We utilize the virtual server answers for guarantee information consistency by Amazon Dynamo (Liu \& Lai, 2018). Dynamo's apportioning plan depends on an assortment of reliable hashing. In their plan, the subsequent range or space of a hash work is considered as a ring. Each individual from the ring is a virtual hub (have) where a physical hub might be in charge of at least one virtual hubs. The presentation of virtual hubs, rather than utilizing settled physical hubs on the ring, is a decision that gives better accessibility and burdens adjusting under disappointments. Every datum thing can be relegated to a hub on the ring dependent on its key. The hashed estimation of the key decides its situation on the ring. Information at that point is allocated to the nearest hub on the ring clockwise. In addition, information is recreated on the progressive $\mathrm{K} 1$ hubs for a given replication factor $\mathrm{K}$, maintaining a strategic distance from virtual hubs that have a place with the equivalent physical hubs. Every one of the hubs on Dynamo is viewed as equivalent and can figure the reference list for some random key. The reference list is the rundown of hubs that store a duplicate of information referenced by the key. Dynamo is an in the end predictable framework. Updates are no concurrently engendered to imitations. Be that as it may, in this paper, we have proposed answers for virtual servers to work all the more proficiently, in this manner improving the effectiveness of the outline to guarantee steady information. Specifically, predicting the distributed systems with least completion time makes a complex commercial decision in resource allocation and scheduling. The methods of detecting deadlock using two way search algorithms help to enhance the productivity of resources assigned in different platform. In this manuscript, the authors propose a novel solution that uses virtual servers to update the replicas.

Specific contributions are:

1) It is a related asset allotment arrangement which empowers discovery and taking care of stops for virtual servers.

2) Conduct tests and thinks about to demonstrate the adequacy of the new proposition.

\section{RELATED WORKS}

This Section manages the related examination and foundation work. Yadgar, Kolosov, Aktas and Soljanin, E. (2019), the issue of parceling online interface diagram for web positioning in peer-to-peer is detailed to be negligible cut set having thickness adjusted dividing. 


\section{The Method Balance Consistency Availability on Systems Virtual Machine}

The issue is ended up being a nondeterministic polynomial hard, by lessening to the base bi area issue (Shen, Guoxin \& Harrison ,2015) ( Shen, Liu \& Chandler ,2015). In peer-to-peer based Page natural companions in P2P organizing frameworks. Peer-to-peer are segregated Rank Davide, Andrea, Stefano , Michele and Giuseppe (2015), Singh and Bawa (2018), Jamal and Butt (2017), every calculation companion consist of a neighborhood web-connect diagram and its Page Rank is processed locally. Peer-to-peer is a suitable decision to be taken care with such confinement. To have the capacity to figure the worldwide positioning, an exceptional hub, supposed word-hub, is developed to store the connection page data of alternate associates. In Fariborz, N. (2016), the creators advance for verification between with two different inlay systems as structured and unstructured.

Nakashima, T and Satoshi, F. (2013), proposed a way to deal with fabricate a tree by recursively choosing an agent and parceling strategy. With this strategy, it underpins just to store data of the tree structure in their sub-space for all the halfway refresh the framework applications with various issues, the procedure includes pointless overhead which is being made amid keeping up the tree from hub disappointments. The frameworks can't check blunder from heterogeneous hubs while expanding message ask for refresh (Liu \& Lai, 2018). The technique is intended for building the tree structure scattering by just including heterogeneous hubs. In this paper, creators are occupied with strategy upkeep and refresh spread. Consequently, the issue likewise proficiently assembles the tree structure dispersal and parallel tree disintegration. In this paper, with the end goal to make it adjusted and hearty, the techniques measurably upgraded will proposed in area 4. In Nguyen and Doan (2017), Vivek introduced two sorts limited consistency are furnished with talk spreading and imitation chain. They have utilized this way to deal with guarantee a specific likelihood of another variant which is being gotten in unstructured peer-to-peer frameworks. The likelihood is tuned by altering the excess degree in spreading a refresh to keep up the harmony between the interchanges overhead with the consistency strictness. The message communicate is an unstructured byte exhibit that is conveyed to all individuals from the gathering through the strategy probabilistic limited consistency. In the past study Nguyen and Doan (2017), technique usually used for getting right mathematical answer in developing unstructured peer-to-peer charts reasonable for varied arbitrary determination. Liu and Lai (2018), expands SCOPE by making both chart development and inquiry goals touchy to hub limits. Different models of unstructured chart development plans incorporate Araneola, a methodology by Law and Siu, and Jianming Fu et al. None of these consider hub heterogeneity. Malecki (2016), the portrayed system can be utilized as arbitrary hub choice crude, yet similar to the case with the recently made reference to plans, does not consider hub heterogeneity.

In $\mathrm{P} 2 \mathrm{P}$ frameworks, to maintain a strategic distance from struggle movement choices in successive hubs, it will contact the reserved ancestors. With the technique, the frameworks hubs. In this way, the strategy turns out to be exceptionally trouble refresh tree structure and it cannot be occupied with the question are in the protest's refresh spread tree. When we

$\mathrm{P} 2 \mathrm{P}$ in conveyed framework are to recover the lost bundles. In Bermbach and Jorn (2013), proposed show is portrayed for consistency support. Furthermore, in Nakashima, $\mathrm{T}$ and Satoshi, F. (2013), it is reached out by a consistency display for $\mathrm{P} 2 \mathrm{P}$ applications. The utilized strategies are half and half of push and force looking after consistency. By Addition, they have likewise used to give application customized reserve consistency, albeit every hub can indicate its consistency prerequisite. In paper Shen, Guoxin and Harrison (2015), the model makes every hub play out the most grounded consistency upkeep from all its relative hubs in the overlay reproduction chain of importance. The techniques introduced are intended for keeping up consistency at a hub which can't be lessened even. Still, we can see that a large portion of the papers have not concentrated working on it investigation of solid consistency. In this paper, we have demonstrated the arrangement strategy to refresh consistency in this above setting.

\section{ALLOCATION OF RESOURCE DISTRIBUTED ON HETEROGENEOUS PLATFORMS}

The server based PC, a few equipment and programming assets are accessible for being arranged. Subsequently assignment of assets ought to be in a proficient way with the goal that we may use the assets in an ideal manner.

Asset allotment: Throughout the years, the model put together registering based with respect to cloud has made parcel of consideration of analysts around the globe with Cloud clients. The Cloud based registering delineates a model of asset distribution other than network or planning. Especially, Amazon C2 is capable of allocating smaller computing resources, rather than a few, large requirements. The traditional distributed computing systems with totally separate architecture competing with cloud based heterogeneity.

No same with the distribution system traditional, we can see a system does not even include a file that is too connected by a network transport. Slow delay in transactions are a limit but not guess before (Yadgar, Kolosov, Aktas \& Soljanin ,2019) (Bermbach \& Jorn , 2013) (Nakashima \& Satoshi , 2013).

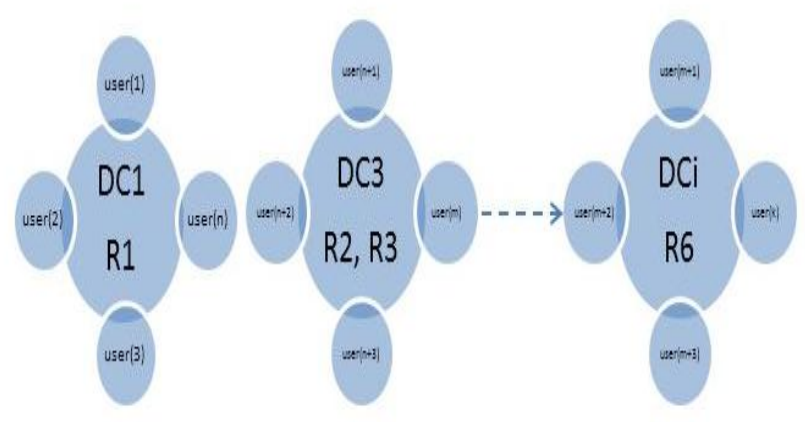

Figure 1.describes many replicas in cloud storage systems. 


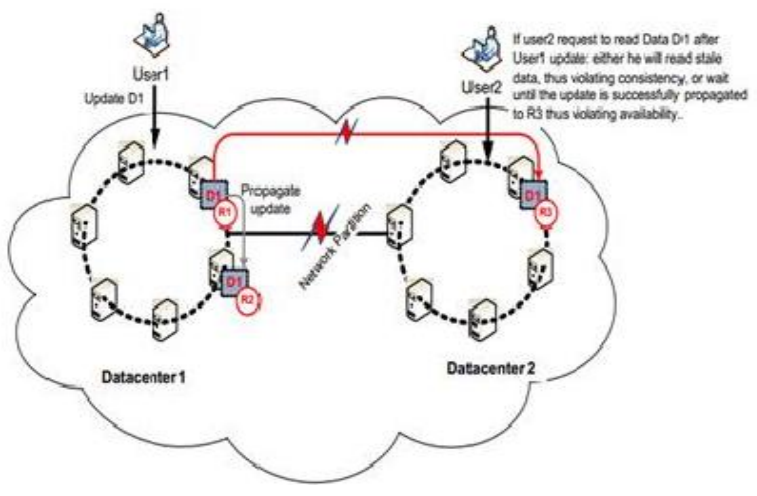

Figure 2 A requirement of data consistency.

We have used the platform graph, for the grid platform. We model a collection of heterogeneous resources and the communication links between them as the nodes and edges of an undirected graph. See an example in Figure 2 A requirement of data consistency.with simple platform on Industry 4.0. In Industry 4.0 is indispensable are the following virtual machines providing services.

\section{RESOURCES ALLOCATION FOR VIRTUAL SERVERS ON THE DATA CONSISTENCY MAINTENANCE}

As presented above, ensuring data consistency, the virtual servers are responsible for propagating updates to replicas. In addition, the virtual server is responsible for processing any other requests from the user. Inside, scale system is not stable, the heterogeneous user: the ability to process, bandwidth usage, the churn rate, the update rate. So, resource allocation (RAM, CPU) for the virtual server is very important. They need to be appropriate. Moreover, solving other problems as deadlock will improve the efficiency of the virtual server in the consistency maintenance scheme. In the model with cloud computing as presented over, the assets gave are accumulated in such a large number of muddled advances. The improvement of an answer for avoid halt need to guarantee that something like one of the accompanying conditions can't happen. Model with cloud computing as presented over, the assets gave is assembled in such a significant number of confused advances. The advancement of an answer for anticipating gridlock need to guarantee that no less than one of the accompanying conditions can't happen: Resources can't be shared, possessed and the extra assets required, no recuperation assets, presence of any cycle or not.

Error! Reference source not found. portrays issues occasion with two hubs and one administration, showing asset allotments potential outcomes.
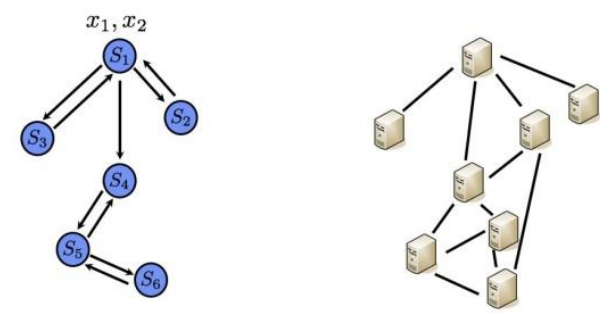

Figure 3.Example problem instance with two nodes and one service, showing possible resource allocations.

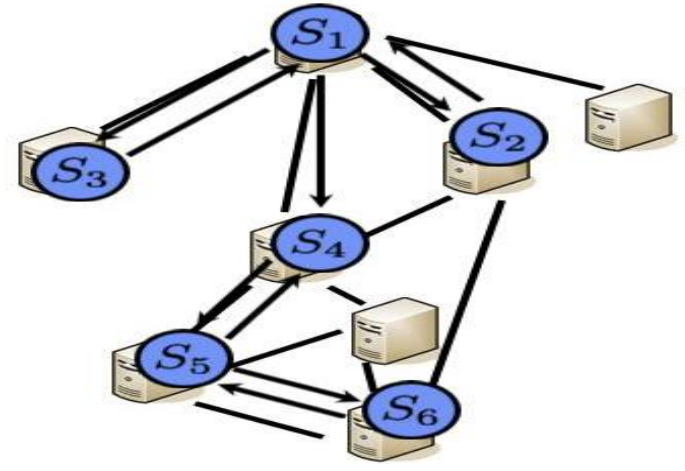

The proposed calculation demands asset portion virtual machines with Peer - To - Peer virtual host conveyance on physical hub at once point. To characterize the dispersion of the majority of a virtual host on the physical hubs in the time required, beginning from time $\mathrm{t}$ and drag long $\mathrm{d}$ seconds is extremely troublesome. As with combine and force and best algorithm, we got the previous time and the algorithm used to provide the resource in the shared environment, because they don't be up the calendar and requires best. Right when the center points have been masterminded, the model uses the best effort computation to scatter all VM's.

Previously mentioned calculation can distribute numerous VM's on a similar hub. With this exploration we are expecting to give proficient circulation of assets, we have proposed the accompanying strategy in disseminated conditions. For this situation, the calculation attempts to convey however many VM's as would be prudent on various physical hubs.

A. Resources Allocation for virtual servers on the data consistency maintenance

In this paper, the viable ramifications direct that architects decide on best exertion accessibility, subsequently ensuring consistency, and covetous consistency for frameworks that must ensure accessibility (Rimal \& Gochhait, 2019). With an even minded approach to deal with the tradeoff is by adjusting consistency accessibility tradeoff in frameworks as shown in Figure 3.Example problem instance with two nodes and one service, showing possible resource allocations..

\section{RESULT ANALYSIS}

This segment manages the outcome examination and exchange. To do as such, the arrangement incorporates two stages: Initially, Making of organized tree; Besides, checking if cycle exists in that tree. In the initial step multicast to be utilized in dDT calculation development so as to send solicitation to on the hubs in the frameworks and sitting tight for answers from them. After this procedure, we get a tree. In the subsequent advance, another calculation to be utilized so as to identify cycle. Be that as it may, this calculation has not been referenced in this paper.

\section{CONCLUSION}

In the substance of the article, we give Open Stack virtual server arrangement. The arrangement concerns the status criteria since it influences the expense of setting up the foundation. 


\section{The Method Balance Consistency Availability on Systems Virtual Machine}

Virtualization arrangements dependent on Open Stack can possibly address the issues of the clients with regards to perplexing and complex wise processing frameworks.

\section{REFERENCES}

1. Malecki, E. J. (2016). Hub Cities in the Evolving Internet. In Hub Cities in the Knowledge Economy (pp. 139-160). Routledge.

2. Yadgar, G., Kolosov, O., Aktas, M. F., \& Soljanin, E. (2019). Modeling The Edge: Peer-to-Peer Reincarnated. In 2nd \{USENIX\} Workshop on Hot Topics in Edge Computing (HotEdge 19).

3. Bermbach, D, and Jorn, K. (2013), "Consistency in distributed storage systems", Networked Systems, Springer Berlin Heidelberg, pp.175-189, 2013.

4. Liu, Y. A., Stoller, S. D., \& Lin, B. (2017). From clarity to efficiency for distributed algorithms. ACM Transactions on Programming Languages and Systems (TOPLAS), 39(3), 12.

5. Nakashima, T and Satoshi, F. (2013), "Tree-Based Consistency Maintenance Scheme for Peer-to-Peer File Sharing Systems", Computing and (CANDAR), 2013 First Interna-tional Symposium on IEEE, pp.187-193, 2013.

6. Shen, H., Guoxin L., and Harrison, C. (2015), "Swarm intelligence based file replication and consistency maintenance in structured P2P file sharing systems", IEEE Transactions on Computers, pp.2953-2967, 2015.

7. Shen, H., Liu, G., \& Chandler, H. (2015). Swarm intelligence based file replication and consistency maintenance in structured P2P file sharing systems. IEEE Transactions on Computers, 64(10), 2953-2967.

8. Fariborz, N. (2016), "Toward authentication between familiar Peers in P2P networking systems"', Autonomous Systems 2016, Proceeding of the 9th GI conference, pp.88-103, 2016.

9. Nguyen, H. H. C., Nguyen, H. M., \& Doan, T. S. (2017). The Method of Maintaining Data Consistency in Allocating Resources for the P2P Network Model. In Context-Aware Systems and Applications, and Nature of Computation and Communication (pp. 155-165). Springer, Cham.

10. Liu, C. M., \& Lai, C. C. (2018). A group-based data-driven approach for data synchronization in unstructured mobile P2P systems. Wireless Networks, 24(7), 2465-2482.

11. Nguyen, H. H. C., Nguyen, H. M., \& Doan, T. S. (2017). The Method of Maintaining Data Consistency in Allocating Resources for the P2P Network Model. In Context-Aware Systems and Applications, and Nature of Computation and Communication (pp. 155-165). Springer, Cham.

12. Davide, A., Andrea, G., Stefano, G., Michele, P., Giuseppe P. (2015). A Fuzzy Logic approach for Re-sources Allocation in Cloud Data Center. Proceedings 2015 IEEE Globecom Workshops (GC Wkshps). pp.1-6, 2015 .

13. Singh, H. J., \& Bawa, S. (2018). Scalable Metadata Management Techniques for Ultra-Large Distributed Storage Systems--A Systematic Review. ACM Computing Surveys (CSUR), 51(4), 82.

14. Jamal, T., \& Butt, S. A. (2017). Cooperative Cloudlet for Pervasive Networks. Proc. of Asia Pacific Journal of Multidisciplinary Research, 5(3), 42-26.

15. Butt, S. A. (2016). Study of agile methodology with the cloud. Pacific Science Review B: Humanities and Social Sciences, 2(1), 22-28.

16. Butt, S. A., Tariq, M. I., Jamal, T., Ali, A., Martinez, J. L. D., \& De-La-Hoz-Franco, E. (2019). Predictive Variables for Agile Development Merging Cloud Computing Services. IEEE Access, 7, 99273-99282.

17. Butt, S. A., Abbas, S. A., \& Ahsan, M. (2016). Software Development Life Cycle \& Software Quality Measuring Types. Asian Journal of Mathematics and Computer Research, 112-122.

18. Rimal,Y., \& Gochhait, S. (2019). Neural Network Machine Learning Analysis for Noisy Data: R Programming International Journal of Engineering and Advanced Technology (IJEAT) ISSN: 2249 - 8958, Volume-8 Issue-6, August 2019.

\section{AUTHORS PROFILE}

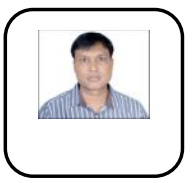

Saikat Gochhiat, Post Doctoral Fellow from University of Extremadura, Spain. Awarded with MOFA Taiwan Fellowship. He has more than 50 publications in journals indexed in Scopus, ABDC, Google Scholar etc.

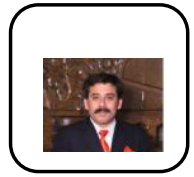

Shariq Aziz Butt received the master's degree from Superior University, Lahore, Pakistan in 2016 andPhD degree from The University of Lahore, Pakistan.

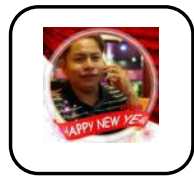

Nguyen ha Huy Cuong, The University of Danang, College of Information Technology, Da Nang, Viet Nam 